J. Electroanal. Chem., 287 (1990) 1-12

Elsevier Sequoia S.A., Lausanne - Printed in The Netherlands

\title{
An inversion method based on information theory applied to quantitative analysis of complex H-adatom electrodesorption voltammograms
}

\section{A.J. Arvia and E. Custidiano}

Instituso de Investigaciones Fisicoqulmicas Teóricas y Aplicadas (INIFTA), Faculaad de Ciencias Exactas, Universidad Nacional de La Plata, Sucursal 4, Casilla de Correo 16, 1900 La Plata (Argentina)

\section{A. Plastino and L. Rebollo Neira}

Departamento de Fisica, Universidad Nacional de La Plata, Casilla de Correo 67, 1900 La Plata (Argentina) (Received 26 September 1988; in revised form 18 January 1990)

\section{ABSTRACT}

An inversion method based on information theory is employed to describe the H-adatom electrodesorption voltammogram resulting for different Pt electrodes in an acid electrolyte. This method can predict exactly the entire behaviour of the voltammograms from only a few points selected from the experiment.

\section{INTRODUCTION}

Mathematical processes of inversion are extremely useful for solving the components of an unknown spectrum. Here the term spectrum should be understood to include one-dimensional data from experiments that do not explicitly involve optical phenomena. A selection of the most commonly used inversion techniques is given in refs. 1-3.

In electrochemistry the linear potential sweep technique, either as a single or a triangular sweep, applied to an electrode where a surface electrochemical reaction takes place [4-8], produces a current-potential profile (voltammogram) which is characterized by current peaks located at potentials (relative adsorption energies) which are specific for each adsorbate and its environment. The voltammogram provides information about the kinetics and some structural aspects of those processes concerning the adsorbate on a particular substrate. The area of the current peaks, corresponding to either adsorbate electroformation or stripping, gives the amount of adsorbate present at the substrate. The amount of adsorbate detectable 
ranges from a small fraction of monolayer upwards. Hence, as for gas-phase reactions at metal surfaces, one should expect that for a particular substrate, the influence of the crystallographic faces on the adsorbate-substrate interaction will also be reflected in the voltammogram. This seems to be the case for many reactions taking place at different single-crystal $\mathrm{Pt}$ surfaces, such as the $\mathrm{H}$-adatom electroadsorption and electrodesorption reactions in acid electrolytes [9-18].

The linear potential sweep technique offers a very simple way to follow changes in the adsorbate-substrate interactions which can be attributed to modifications in the substrate surface morphology. The question now is to what extent can these relative changes be determined quantitatively through an adequate and precise numerical analysis of the voltammogram considered in this case as an electrochemical spectrum.

Quantitative analysis of the distribution of the $\mathrm{H}$-adatom adsorption states at $\mathbf{P t}$ electrodes have been presented by several authors by considering either a single complex relationship between the adsorption energy and adsorbate coverage which accounts for the potential dependence of the adsorbate surface coverage, or by assigning to each distinguishable adsorption site a specific adsorption isotherm [19-27].

The present work describes the application of a powerful and successfully proved inversion method based on the information theory (IT) [28-31] to electrochemical spectra. For this purpose a certain number of adsorbate states are selected according to the number of voltammetric peaks, and for each adsorbate, a general electrochemical adsorption isotherm with an interaction parameter varying within the potential range of the adsorption reaction is considered [32]. The inversion method can predict exactly the entire behaviour of complex voltammetric profiles from only a very small number of experimental points.

\section{CALCULATION PROCEDURE}

\section{The electrochemical formalism}

Let us consider the $\mathrm{H}$-adatom electroadsorption process at equilibrium in acid solution on the $N$-site of the metal surface, $\mathrm{Me}_{N},\left(\mathrm{Me}_{N}=\mathrm{Pt}_{N}\right)$ :

$\left(\mathrm{e}^{-}\right) \mathrm{Me}_{n}\left(\mathrm{H}_{2} \mathrm{O}\right.$, ions $)+\mathrm{H}_{\text {(i) }}^{+}(\mathrm{aq}) \rightleftharpoons \mathrm{Me}_{N}\left(\mathrm{H}, \mathrm{H}_{2} \mathrm{O}\right.$, ions $)$

Reaction (1) implies as reactant two ensembles, namely $\left(\mathrm{e}^{-}\right) \mathrm{Me}_{N}\left(\mathrm{H}_{2} \mathrm{O}\right.$, ions) and $\mathrm{H}_{(\mathrm{i})}^{+}(\mathrm{aq})$, where $N$ denotes a distinguishable site at the metal surface, $\mathrm{Me}$, and $\mathrm{H}_{(\mathrm{i})}^{+}(\mathrm{aq})$ refers to the aquated proton in the inner part of the electrical double layer. Likewise, $\mathrm{Me}_{N}\left(\mathrm{H}, \mathrm{H}_{2} \mathrm{O}\right.$, ions) represents the reaction product. The idea of conceiving the adsorbate in terms of a complex potential-dependent ensemble for $\mathrm{H}$-adatom adsorption on metal electrodes was recently discussed to interpret the kinetic data for this reaction [33]. Thence, the characterististics of each ensemble come from both collective effects involving the crystallographic features of $\mathbf{M e}_{N}$ and the interaction energy with solvent molecules (water) and ions (anions and cations) in 
solution. For the sake of simplicity, let us further consider that neither double-layer effects nor transport processes at the solution side play a significant role in the electrochemical reaction. Under these conditions, the instantaneous current density $j(t)$ is given by the expression [5,34].

$$
\begin{aligned}
j(t)=q_{\mathrm{m}} \frac{\mathrm{d} \theta}{\mathrm{d} t}= & q_{\mathrm{m}}\left[k_{\mathrm{f}}^{\prime} c_{\mathrm{H}^{+}}(1-\theta) \exp [(1-\alpha) E F / R T] \exp [-(1-\alpha) r \theta]\right. \\
& -k_{\mathrm{b}} \theta \exp (-\alpha E F / R T) \exp (\alpha r \theta)
\end{aligned}
$$

where $q_{\mathrm{m}}$ is the charge density required to form a monolayer of $\mathrm{H}$ adatoms; $\theta$ is the degree of surface coverage by $\mathrm{H}$ atoms; $k_{\mathrm{f}}^{\prime}$ and $k_{\mathrm{b}}$ denote the rate constants for the forward and backward reactions, respectively, referred to the standard hydrogen electrode potential; $c_{\mathrm{H}^{+}}$is the hydrogen ion concentration in the bulk solution; $E$ is the potential applied to the electrode; and $r$ (in $R T$. units) is a variable correction parameter included in the electrochemical adsorption isotherm for the $\mathrm{H}$ adatoms. The parameter $r$ is taken as a constant in the Temkin isotherm, and it has been interpreted in terms of the change in the adsorption Gibbs energy due to either intrinsic surface heterogeneity or lateral interaction for the adsorbate species or of induced heterogeneity at the substrate surface [35]; $F, R$ and $T$ have their usual meanings.

The adsorption isotherm for the adsorbate comes from eqn. (2) under quasi-equilibrium conditions, i.e. $j(t)=0$. Then Frumkin's isotherm results:

$$
\frac{(1-\theta)}{\theta}=K(N) \exp (-E F / R T) \exp (r \theta)
$$

where

$K(N)=\frac{k_{\mathrm{f}}^{\prime}(N) c_{\mathrm{H}^{+}}}{k_{\mathrm{b}}(N)}=\frac{k_{\mathrm{f}}(N)}{k_{\mathrm{b}}(N)}$

The value of $j(t)$ can be expressed in terms of the variation of surface coverage, $\theta$, with time, as follows:

$j(t)=q_{\mathrm{m}} \frac{\mathrm{d} \theta}{\mathrm{d} t}=C \frac{\theta(1-\theta)}{[1+r \theta(1-\theta)]}$

where $C$ is a constant.

Let us now assume that each distinguishable reacting ensemble on the substrate can be assigned to a different value of $N(N=1,2,3,4,5)$, so that $K(N)$, the equilibrium constant of reaction (1), changes for each reacting ensemble. Therefore, if $K(1)$ represents the particular value of the specific rate constant at site $N=1$; then $\theta(1, r, E)$ should be the solution of eqn. (3) for $K(N)=K(1)$, provided that $\left[r_{1}, r_{2}\right]_{1}$ is the interval of variation of $r$ for site $N=1$. Therefore, eqn. (5) can be put forward in a more general way as follows:

$j(t)=q_{\mathrm{m}} \frac{\mathrm{d} \theta}{\mathrm{d} t}=C \frac{\theta(N, r, E)[1-\theta(N, r, E)]}{\{1+r \theta(N, r, E)[1-\theta(N, r, E)]\}}$ 
The equilibrium constant $K(N)$ involved in eqn. (3) can be determined from the maximum of $j(t)$ predicted by eqn. (6) for a certain potential value associated with $j(t)$. Accordingly, the expression for $K(N)$ results:

$K(N)=\exp \left(-E_{N} F / R T\right) \exp (r / 2)=K^{\circ}(N) \exp (r / 2)$

where $E_{N}$, the potential related to $K(N)$, is the voltammetric peak potential. $K^{\circ}(N)$ is the equilibrium constant for reaction (1) for $r=0$, that is, when the Langmuir adsorption isotherm conditions are fulfilled [5,34].

Let us further assume that the overall voltammogram of $\mathrm{H}$-adatom electrodesorption results from the overlapping of specific voltammograms related to the different reacting ensembles, and let us also admit that the interaction parameter for each ensemble changes according to a certain distribution function, $\rho(N, r)$. Hence, the equation for the overall voltammogram can be finally written as follows:

$\langle j(E)\rangle=A \sum_{N=1}^{N_{1}} \int_{r_{1}}^{r_{2}} \rho(N, r) \frac{\mathrm{d} \theta(N, r, E)}{\mathrm{d} t} \mathrm{~d} r=I(E)$

where $A$ denotes an overall constant. This equation exhibits clearly the main features of the present model. For a given value of $E$, the value of $j(E)$ is expressed as a mean value, i.e. measurements allow one to ascertain $j(E)$ only in the form of a statistical average.

The inversion method

Let us consider a set of experimentally determined values $I\left(E_{i}\right), i=1, \ldots, M$. For each $i$, eqn. (8) reads

$I\left(E_{i}\right)=A \sum_{N=1}^{N_{i}} \int_{r_{1}}^{r_{2}} \rho(N, r) \frac{\mathrm{d} \theta}{\mathrm{d} t}\left(N, r, E_{i}\right) \mathrm{d} r \quad i=1, \ldots, M$

Out of these $M$ equations, let us select one of them, for instance the one corresponding to $i=1$, in order to fix the value of the constant $A$ :

$$
A=\frac{I\left(E_{1}\right)}{\sum_{N=1}^{N_{\mathrm{t}}} \int_{r_{1}}^{r_{2}} \rho(N, r) \frac{\mathrm{d} \theta}{\mathrm{d} t}\left(N, r, E_{1}\right) \mathrm{d} r}
$$

After substituting eqn. (10) into eqn. (9) one obtains:

$$
I\left(E_{i}\right)=\frac{I\left(E_{1}\right) \sum_{N=1}^{N_{i}} \int_{r_{1}}^{r_{2}} \rho(N, r) \frac{\mathrm{d} \theta}{\mathrm{d} t}\left(N, r, E_{i}\right) \mathrm{d} r}{\sum_{N=1}^{N_{t}} \int_{r_{1}}^{r_{2}} \rho(N, r) \frac{\mathrm{d} \theta}{\mathrm{d} t}\left(N, r, E_{1}\right) \mathrm{d} r}
$$


Equation (11) can be written in the following way:

$$
\begin{aligned}
& I\left(E_{i}\right) \sum_{N=1}^{N_{\mathrm{t}}} \int_{r_{1}}^{r_{2}} \rho(N, r) \frac{\mathrm{d} \theta}{\mathrm{d} t}\left(N, r, E_{1}\right) \mathrm{d} r \\
& =I\left(E_{1}\right) \sum_{N=1}^{N_{\mathrm{t}}} \int_{r_{1}}^{r_{2}} \rho(N, r) \frac{\mathrm{d} \theta}{\mathrm{d} t}\left(N, r, E_{\mathrm{i}}\right) \mathrm{d} r
\end{aligned}
$$

or as the following simplified expression:

$$
\sum_{N=1}^{N_{i}} \int_{r_{1}}^{r_{2}} \rho(N, r) \sigma_{i}(N, r) \mathrm{d} r=0 \quad i=2, \ldots, M
$$

where

$$
\sigma_{i}(N, r)=I\left(E_{i}\right) \frac{\mathrm{d} \theta}{\mathrm{d} t}\left(N, r, E_{1}\right)-I\left(E_{1}\right) \frac{\mathrm{d} \theta}{\mathrm{d} t}\left(N, r, E_{i}\right)
$$

The mathematical task is just that of inverting the system of eqns. (13). However, this problem has, in general, several different solutions. In other words, a variety of functional forms $\rho(N, r)$ will be able to reproduce $I\left(E_{i}\right)$ data correctly. The problem is to establish the basis for selecting a unique solution. It is known that the correct answer for this type of problem, in which a solution is to be selected from a given set, is given by IT [28-31]. It provides a definite criterion for building up such a unique solution by recourse to the maximum entropy principle, which asserts that out of all the available solutions there is only one which maximizes the function $S$, denoted as the "entropy", as given by the equation:

$S=-\sum_{N=1}^{N_{t}} \int_{r_{1}}^{r_{2}} \rho(N, r) \ln \rho(N, r) \mathrm{d} r$

It should be noted that $S$ is not necessarily related to the entropy definition in thermodynamics [28-31]. As is shown elsewhere [36], the adequate solution should be of the form

$\rho(N, r)=\exp \left(\lambda_{1}\right) \exp \left(\sum_{i=2}^{M} \lambda_{i} \sigma_{i}(N, r)\right)$

where the $\lambda$ 's are Lagrange multipliers. The normalization condition

$$
\sum_{N=1}^{N_{1}} \int_{r_{1}}^{r_{2}} \rho(N, r) \mathrm{d} r=1
$$

allows for an explicit analytical expression for $\lambda_{1}$ :

$\lambda_{1}=-\ln \sum_{N=1}^{N_{t}} \int_{r_{1}}^{r_{2}} \exp \left[-\sum_{i=2}^{M} \lambda_{1} \sigma_{i}(N, r)\right] \mathrm{d} r$

The remaining $(M-1)$ Lagrange multipliers are easily obtained by recourse to any appropriate iterative algorithm, such as Newton's [37]. 
Let us call $\rho^{m}(N, r)$ the solution obtained by following the path described above on the basis of $m$ pieces of data $(m<M)$. Now the function $\rho^{m}$ can be employed to "predict" values of $I\left(E_{i}\right)$ for $i>m$. Let us denote these "predictions" by $I^{m}(E)$, expressed in the following way:

$I^{m}(E)=A \sum_{N=1}^{N_{1}} \int_{r_{1}}^{r_{2}} \rho^{m}(N, r) \frac{\mathrm{d} \theta}{\mathrm{d} t}(N, r, E) \mathrm{d} r$

If these "predictions" agree within experimental error with the measured values, then $\rho^{m}(N, r)$ can be considered as a good representation of the "true" $\rho(N, r)$ function. Otherwise, if the agreement is poor, then the value of $m$ should be changed systematically to obtain a satisfactory representation. It is well established that this approach always converges [31].

In the following section it will be seen that a rather small set of $m$ data suffices to predict a large number of voltammograms correctly. Indeed, taking $m=8$ allows an excellent representation of the experimental data.

When a suitable representation for $\rho(N, r)$ is obtained, one can evaluate the relative percentages, $P(N)$, assigned to each type of site, $N$, through the following relationship:

$$
P(N)=\frac{\int_{r_{1}}^{r_{2}} \rho(N, r) \mathrm{d} r}{\sum_{N=1}^{N_{1}} \int_{r_{1}}^{r_{2}} \rho(N, r) \mathrm{d} r} \quad N=1,2, \ldots, N_{\mathrm{t}}
$$

and, correspondingly, the appropriate interaction parameters:

$$
\langle r(N)\rangle=\frac{\int_{r_{1}}^{r_{2}} r \rho(N, r) \mathrm{d} r}{\int_{r_{1}}^{r_{2}} \rho(N, r) \mathrm{d} r} \quad N=1,2, \ldots, N_{\mathrm{t}}
$$

\section{RESULTS AND CONCLUSIONS}

The inversion method described above was employed specifically for the quantitative analysis of voltammograms obtained at different linear potential sweep rates, $v$, for the electrodesorption of $\mathbf{H}$ adatoms on different $\mathrm{Pt}$ electrodes - polycrystalline (pc) Pt and Pt with preferred crystallographic orientation (pco), namely (100)type pco Pt and (111)-type pco Pt (Figs. 1 and 2), immersed in $0.5 \mathrm{M} \mathrm{H}_{2} \mathrm{SO}_{4}$ at $25^{\circ} \mathrm{C}$. The denomination of these electrodes is based on the fact that their $\mathrm{H}$-electrodesorption spectra resemble to some extent those reported in the literature for the corresponding single-crystal Pt electrodes under the same experimental conditions [7-18]. 


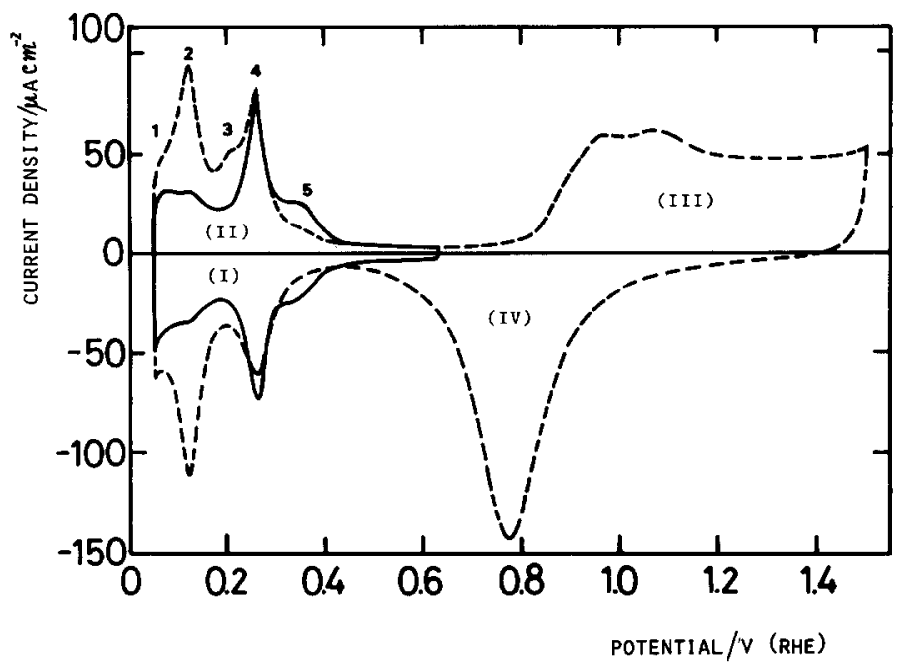

Fig. 1. Current-potential profiles (voltammograms) obtained by applying a triangular potential sweep at $50 \mathrm{mV} / \mathrm{s}$ to platinum electrodes in $0.5 \mathrm{M}$ sulphuric acid solution at $25^{\circ} \mathrm{C}$. The dashed line corresponds to a polycrystalline platinum electrode, $0.20 \mathrm{~cm}^{2}$ geometric area. The full trace corresponds to a (100)-type preferred oriented platinum electrode, $0.15 \mathrm{~cm}^{2}$ geometric area. The potentials are referred to a reversible hydrogen electrode (RHE) in the same electrolyte. Regions I, II, III and IV are related to the $\mathrm{H}$-adatom electroadsorption, the $\mathrm{H}$-adatom electrodesorption, the $\mathrm{O}$-adatom electroadsorption and the O-adatom electrodesorption, respectively. The voltammogram resulting in region I was used for the application of the inversion method derived from IT. The numbers 1, 2, 3, 4 and 5 correspond to $N=1,2,3,4$, and 5 in the text.

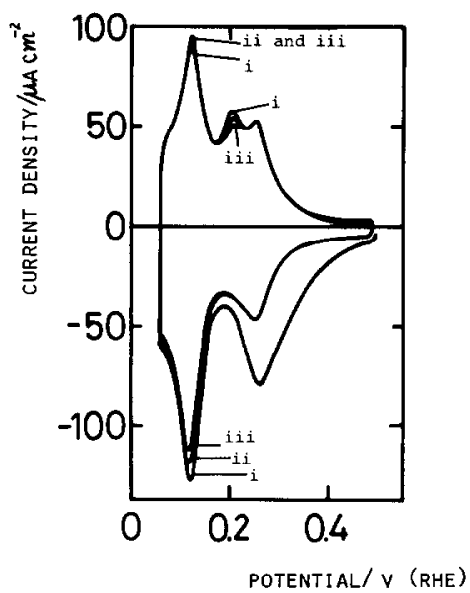

Fig. 2. Current-potential profiles (voltammograms) obtained by applying three successive triangular potential sweeps to a (111)-type pco platinum electrode in $0.5 \mathrm{M}$ sulphuric acid solution at $25^{\circ} \mathrm{C}$. The successive voltammograms arc identified as $i$, ii and iii. The latter corresponds to the stabilized profile and was used for the application of the inversion procedure described in the text. 
TABLE 1

Parameters employed in the calculations ${ }^{\text {a }}$

\begin{tabular}{llllrl}
\hline$N$ & $K(N)$ & $E_{\mathrm{p}} / \mathrm{mV}$ & $\langle r\rangle_{1}$ & $\langle r\rangle_{2}$ & \multicolumn{1}{c}{$\langle r\rangle_{3}$} \\
\hline 1 & $3.31 \times 10^{-2}$ & 87.5 & 0.298 & 0.87 & 0.27 \\
2 & $6.96 \times 10^{-3}$ & 127.5 & -1.41 & 0.85 & -1.62 \\
3 & $3.09 \times 10^{-4}$ & 207.5 & -0.62 & 2.17 & -0.62 \\
4 & $3.17 \times 10^{-5}$ & 262.5 & -0.898 & -1.21 & -0.47 \\
5 & $1.20 \times 10^{-6}$ & 350.0 & -1.9 & 0.90 & -0.78 \\
\hline
\end{tabular}

aalues of $r$ are given in $R T$ units. $R$ is the universal gas constant and $T$ is the absolute temperature.

The H-adatom electrodesorption spectrum for pc $\mathrm{Pt}$ in $0.5 \mathrm{M} \mathrm{H}_{2} \mathrm{SO}_{4}$ at room temperature exhibits at least five electrodesorption peaks identified as 1, 2, 3, 4 and 5 in Fig. 1. Each one of these peaks can be associated with a distinguishable reacting ensemble $(N=1,2,3,4$ and 5). The electrodesorption of each ensemble is characterized by its individual parameter, $K(N)$, calculated at the corresponding peak potential $\left(E_{N}\right)$ and by $\rho(N, r)$. The dependence of the latter on the applied potential changes according to both the type of electrode and $N$. Peaked distribution functions are obtained for $\mathrm{pc} P t$ and (111)-type pco Pt for $N=1$ and $N=3$, and for the three types of Pt electrode for $N=4$, but different dependences resulted for (100)-type pco Pt [38-41] (Figs. 1 and 2). From these complex functionalities, average values of $r$ can be estimated. The five values of $K(N), E_{N}$ and $\langle r\rangle$, the average value of $r$ for the three different $P t$ electrodes, are listed in Table 1, and the distribution of $P(N)$ for the different Pt electrodes is given in Table 2. The construction of the distribution function $\rho(N, r)$ was made with eight experimental points (Fig. 3). The contribution of the electrical double-layer charging along the voltammetric profile was taken as constant for the entire range of the H-electrodesorption reaction. The fit of the experimental and predicted voltammograms for the three types of Pt electrode is certainly very good (Fig. 4a). The fact that the calculation, starting from only a few points, enables us to reproduce the entire H-electrodesorption spectrum so exactly, proves the reliability and power of the calculation procedure.

TABLE 2

Distribution (\%) of $P(N)$ for the different Pt electrodes

\begin{tabular}{llll}
\hline$P(N)$ & $P(N) / \%$ & $P(N) / \%$ & $P(N) / \%$ \\
& pc Pt & $(100)$-type pco Pt & $(111)$-type pco Pt \\
\hline$P(1)$ & 24.15 & 15.7 & 24.7 \\
$P(2)$ & 28.5 & 18.6 & 30.9 \\
$P(3)$ & 16.5 & 4.7 & 19.2 \\
$P(4)$ & 30.6 & 42.8 & 24.7 \\
$P(5)$ & 0.4 & 18.0 & 0.35 \\
\hline
\end{tabular}



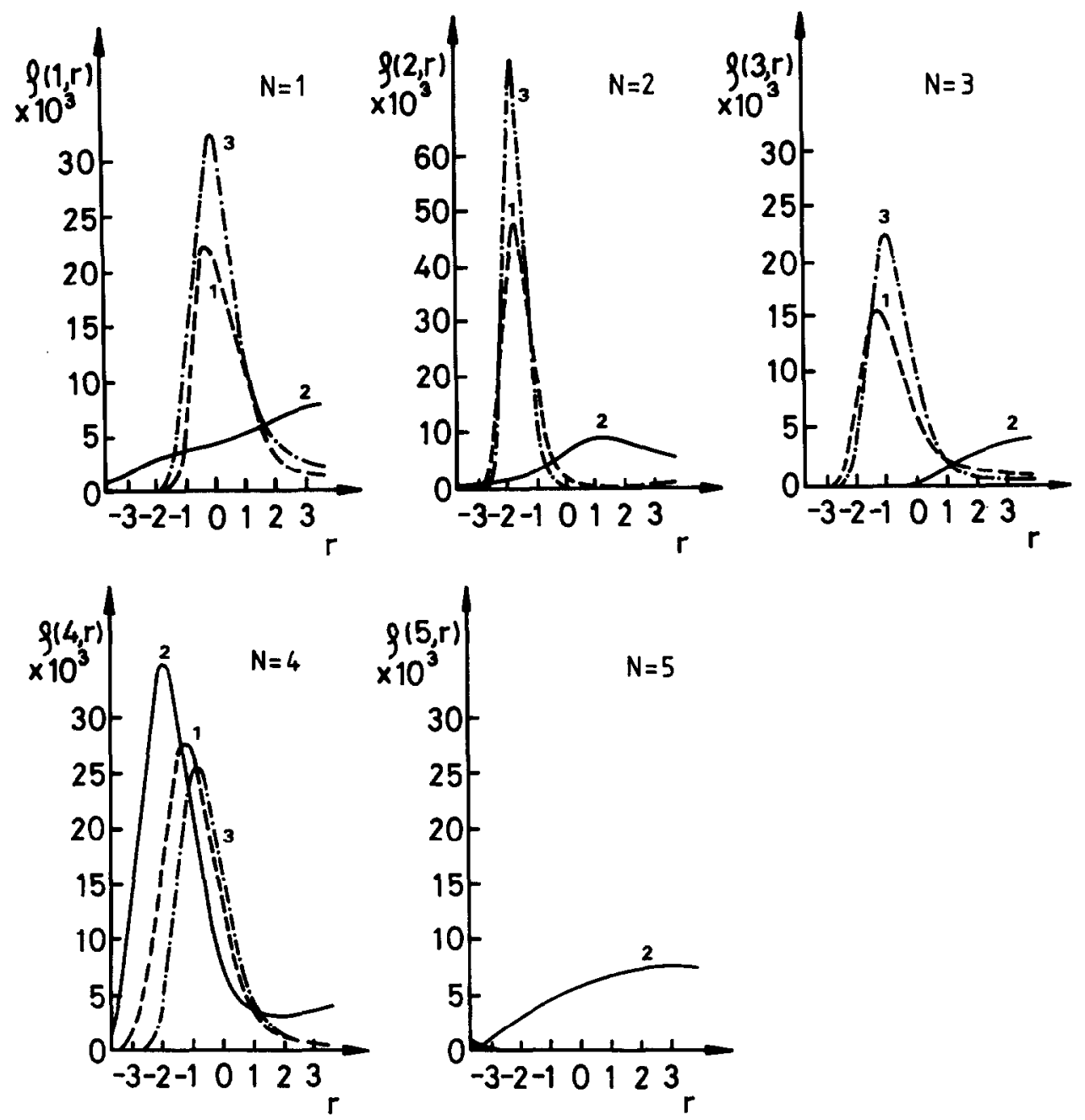

Fig. 3. Distribution functions for $N=1,2,3,4$ and 5. (1) pc Pt; (2) (100)-type pco Pt; (3) (111)-type pco Pt. The values of $r$ are given in $R T$ units.

At this stage, it is interesting to compare the results obtained for $N=1,2,3,4$ and 5 (Fig. 4a) with those for the same number of $N$ and an average $\langle r\rangle$ value resulting from $\rho(r, N)$ for each peak (Fig. 4b), and with those resulting for sites $N=1,2$ and 4 (Fig. 4 c). These results show that the number $N$ of surface sites and the distribution function $\rho(N, r)$ play the most important role in defining the shape of the entire voltammogram. The use of an average value of $r$ for the entire $\mathrm{H}$-electrodesorption reaction furnishes a curve fit comparable to that reported for the distribution of $\mathrm{H}$-adatom states on a $\mathrm{Pt}(100)$ single crystal by means of the MINUIT program [26,27]. 


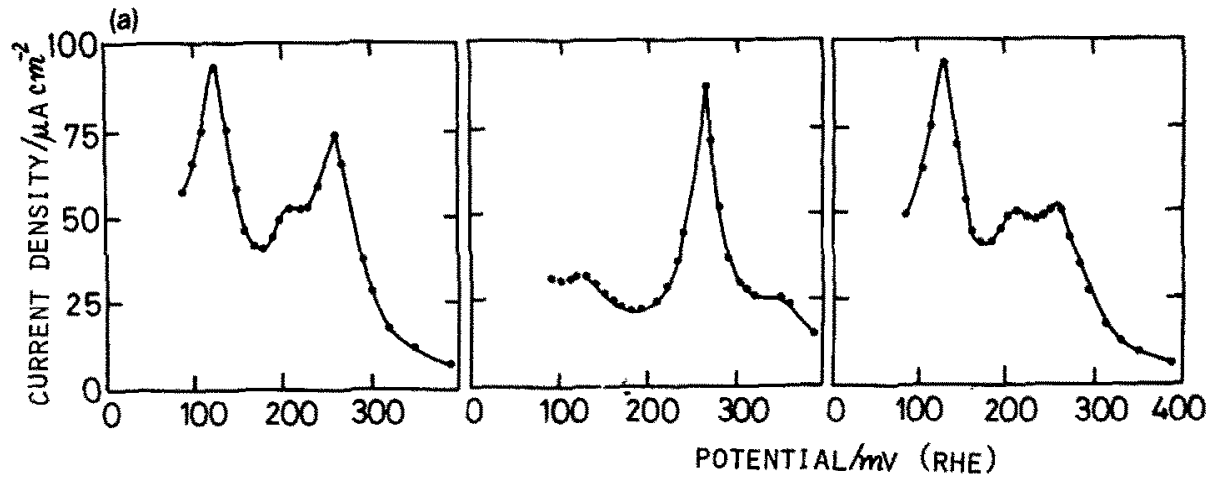

(b)

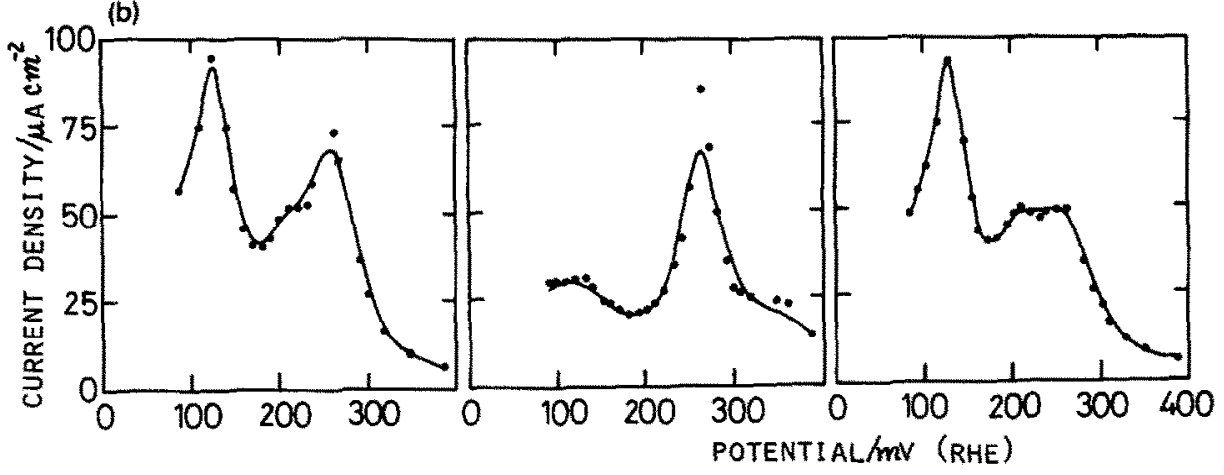

(c)

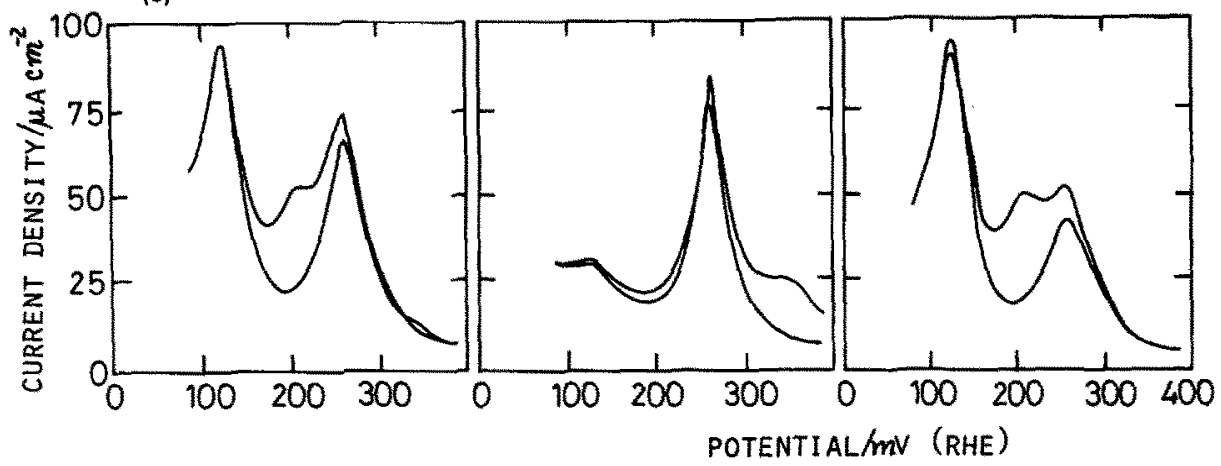

Fig. 4. H-adatom electrodesorption voltammogram for pe Pt, (100)-type pco Pt and (111)-type pco Pt in $0.5 \mathrm{M}$ sulphuric acid at $0.05 \mathrm{~V} / \mathrm{s}, 25^{\circ} \mathrm{C}$. (a) Results obtained for $N=1,2,3,4$ and 5 and $\rho(N, r)$ as given in Fig. 3 (full traces). Experimental data: black points. (b) Results predicted for $N=1,2,3,4$ and 5 and $\langle r\rangle$ values for each $N$ (full trace). Experimental data: black points. (c) Results predicted for different $N$; the corresponding $\rho(N, r)$ is given in Fig. 3. (1) Voltammogram predicted for $N=1,2,3,4$ and 5. (2) The voltammogram predicted for $N=1,2$ and 4 exhibits only the peaks corresponding to $N=1, N=2$ and $N=4$. 
From these results it is interesting to derive the value of $P(N)$ from each value of $N$, as $P(N)$ gives the relative contribution of each adsorbate in the entire potential range of the $\mathrm{H}$-atom electrodesorption reaction. The corresponding data, which are assembled in Table 2, show that the differences in $P(N)$ for the various $\mathrm{Pt}$ electrodes are comparatively smaller than those which might be expected by making a direct assignment of each current peak to a single electroadsorption at a particular crystallographic face. On the other hand, the dependence of $\rho(N, r)$, for each value of $N$, on the relative charge of each adsorbate suggests that the influence of the electrode surface on the electroadsorption process results from adsorbate interactions which depend on the degree of surface coverage and other interaction terms involving the different constituents of the metal-solution interface at the $N$ site.

\section{ACKNOWLEDGEMENTS}

This work was supported financially by the Consejo Nacional de Investigaciones Científicas y Técnicas and the Comisión de Investigaciones Científicas de la Provincia de Buenos Aires.

\section{REFERENCES}

1 A. Tichonov and V. Arsenine, Méthodes de Résolution de Problèmes mal Posés, MIR, Moscow, 1976.

2 J. Albrecht and L. Collatz (Eds.), Numerical Treatment of Integral Equations, Birkhäuser, Basel, 1980.

3 H.S. Hou and H.C. Andrews, IEEE Trans. Comput., 26 (1977) 856.

4 F.G. Will and C.A. Knorr, Z. Elektrochim., 64 (1960) 258.

5 S. Srinivasan and E. Gileadi, Electrochim. Acta, 11 (1962) 321.

6 M.W. Breiter, Trans. Faraday Soc., 62 (1966) 2887.

7 H. Angerstein-Kozlowska, W.B.A. Sharp and B.E. Conway in M.W. Breiter (Ed.), Symposium on Electrocatalysis, The Electrochemical Society, Princeton, NJ, 1974, p. 94.

8 H. Angerstein-Kozlowska and B.E. Conway, J. Electroanal. Chem., 95 (1979) 1.

9 A.S. Homa, E. Yeager and B.D. Cahan, J. Electroanal. Chem., 150 (1983) 181.

10 E. Yeager, W.E. O'Grady, M.Y.V. Woo and P. Hagans, J. Electrochem. Soc., 125 (1978) 346.

11 R.M. Ishikawa and A.T. Hubbard, J. Electroanal. Chem., 69 (1976) 317.

12 A.T. Hubbard, R.M. Ishikawa and J. Kalakaau, J. Electroanal. Chem., 86 (1978) 271.

13 P.N. Ross, J. Electroanal. Chem., 76 (1977) 139.

14 P.N. Ross, Jr., Surf. Sci., 102 (1981) 463.

15 K. Yamamoto, D.M. Kolb, R. Kötz and G. Lehmpfuhl, J. Electroanal. Chem., 96 (1979) 233.

16 J. Clavilier, J. Electroanal. Chem., 107 (1980) 211.

17 J. Clavilier, R. Faure, G. Guinet and R. Durava, J. Electroanal. Chem., 107 (1980) 205.

18 J. Clavilier and D. Armand, J. Electroanal. Chem., 199 (1986) 187.

19 M.W. Breiter, J. Electroanal. Chem., 8 (1964) 449.

20 P. Stonehart, Electrochim. Acta, 15 (1970) 1853.

21 K. Kinoshita, I. Lundquist and P. Stonehart, J. Catal. 31 (1973) 325.

22 K. Kinoshita and P. Stonehart, Electrochim. Acta, 20 (1975) 101.

23 F.E. Woodward, M.K. Hanafey and C.N. Reilley, J. Electroanal. Chem., 167 (1984) 65.

24 D.L. Adams, Surf. Sci., 42 (1974) 12.

25 F.E. Woodward, C.I. Scortichini and C.N. Reilley, J. Electroanal. Chem., 151 (1983) 109.

26 D. Armand and J. Clavilier, J. Electroanal. Chem., 225 (1987) 205. 
27 D. Armand and J. Clavilier, J. Electroanal. Chem., 233 (1987) 251.

28 D.M. Guerin, A. Alvarez, R. Bonetto, A. Plastino and L. Rebollo Neira, Acta Crystallogr., Sect. A, 42 (1986) 30.

29 A. Alvarez, R. Bonetto, D.M. Guerin, A. Plastino and L. Rebollo Neira, Powder Diffraction, 2 (1987) 220.

30 J. Nuñez, L. Rebollo Neira, A. Plastino, R. Bonetto, A.M. Guerin and A. Alvarez, X-Ray Spectrom., 17 (1988) 47.

31 A. Plastino, L. Rebollo Neira and A. Alvarez, Phys. Rev. A, 40 (1989) 1644.

32 S.K. Rangarajan, J. Electroanal. Chem., 82 (1977) 93.

33 S.A. Bilmes, M.C. Giordano and A.J. Arvia, J. Electroanal. Chem., 225 (1987) 183; Can. J. Chem., 66 (1988) 2259.

34 J.M. Hale and R. Greef, Electrochim. Acta, 12 (1967) 1409.

35 P. Delahay, Double Layer and Electrode Kinetics, Interscience, New York, 1965.

36 A. Katz, Principles of Statistical Mechanics, Freeman, New York, 1967.

37 E. Issacson and H.B. Keller, Analysis of Numerical Methods, Wiley, New York, 1966, p. 109.

38 R.M. Cerviño, W.E. Triaca and A.J. Arvia, J. Electroanal. Chem., 182 (1985) 51.

39 J.C. Canullo, W.E. Triaca and A.J. Arvia, J. Electroanal. Chem., 175 (1984) 337.

40 R. Cerviño, W.E. Triaca and A.J. Arvia, Electrochim. Acta, 30 (1985) 10.

41 A.J. Arvia, J.C. Canullo, E. Custidiano, C.L. Perdriel and W.E. Triaca, Electrochim. Acta, 31 (1986) 1359. 